\title{
Intensity difference limens for lingual vibrotactile stimuli
}

\author{
DONALD FUCCI, LARRY H. SMALL, and LINDA PETROSINO \\ School of Hearing and Speech Sciences, Ohio University, Athens, Ohio 45701
}

\begin{abstract}
Lingual differential sensitivity for intensity was measured by determining difference limens (DLs) at three different frequencies and five sensation levels. DLs were obtained from five normal adult subjects. The results indicated a relationship between the DL values (expressed as a Weber fraction: $\Delta \mathrm{I} / \mathrm{I}$ ) and frequency. Overall, the data obtained indicate that the lingual sensory system can provide DL functions that are compatible with those of other tactile sensory systems, as well as those derived from other sensory modalities, such as audition.
\end{abstract}

The measurement of differential sensitivity of both the auditory and tactile sensory systems has previously been examined in order to determine each system's ability to resolve slight differences between perceived stimuli differing in intensity. The difference limen (DL) is the unit typically employed as an index to express the differential sensitivity of a sensory system.

Harris (1963) and Riesz (1928) examined the differential sensitivity of the human auditory system and determined that the DL or "just noticeable difference" (jnd) for intensity is approximately $.50 \mathrm{~dB}$. The results of these investigations also showed that the ability of the auditory system to detect $.50-\mathrm{dB}$ changes in intensity appears to be relatively independent of both sensation level and frequency, except for sensation levels near threshold.

Differential sensitivity for intensity of the tactile sensory system yields somewhat more varied results than those found for the auditory sensory system. The size of the DL (at moderate to high intensities) as measured by various investigators has ranged from .30 (Sherrick, 1950), to .11 (Schiller, 1953), to .05 (Knudsen, 1928). These values were obtained by using the Weber fraction $(\Delta \mathrm{I} / \mathrm{I})$. Craig (1972) suggests that the discrepancies in the reported DL values for tactile sensitivity at moderate to high intensities may be due to differences in the various experimental methodologies. Even though these DL variations exist, Craig (1972) indicates that two generalizations can be made in relation to the factors affecting tactile DLs. First, at low intensities, $\Delta \mathrm{I} / \mathrm{I}$ is large and decreases as intensity increases. Second, DLs for the sense of touch appear to be independent of frequency.

The existing tactile DL information has been obtained primarily from the hand and index finger. The purpose of the present study was to examine the differential vibrotactile sensitivity of the human tongue in order to

The authors would like to acknowledge the contributions of Neal Sloane to the development of this study. determine if it processes DL information in a manner compatible with those structures already examined. More specifically, an attempt was made to establish lingual DL values as related to sensation level and frequency.

\section{METHOD}

\section{Subjects}

Five subjects with no history of speech and/or hearing disorders were selected for this study. All subjects had prior experience with lingual vibrotactile threshold technique. The subjects ranged in age from 22 to 40 years, with a mean age of 29.4 years.

\begin{abstract}
Apparatus
The equipment used in this study has been described in previous publications, the most recent being a chapter on the state of the art (Fucci \& Crary, 1979). The stimulus unit included a sine-wave generator, a frequency counter, an electronic switchinterval timer, an amplifier, a .10-dB step-variable attenuator, and an electromagnetic minivibrator. The pulsed vibratory signal generated had a $50 \%$ duty cycle (on .5 sec and off $.5 \mathrm{sec}$ ) with a rise and decay time of $100 \mathrm{msec}$. The measurement unit consisted of an accelerometer, a cathode follower, a microphone amplifier, and a voltmeter.
\end{abstract}

\section{Procedure}

Each subject was seated in an adjustable chair and asked to place his tongue up against the bottom of a plastic sterilized disk. A hole in the disk provided access of the probe to the anterior midline section of the dorsum of the tongue. The contactor area of the probe was $.128 \mathrm{~cm}^{2}$. To maintain constant pressure of the vibrator on the tongue for all subjects, the probe was lowered until a voltmeter recorded contact. The probe was then lowered $1 \mathrm{~mm}$ into the tongue surface from the point of recorded contact.

Thresholds of lingual vibrotactile sensitivity were first determined for the test frequencies of 125,250 , and $400 \mathrm{~Hz}$. Each subject was required to respond by raising his hand as soon as he detected the pulsed vibratory stimulus on the dorsal lingual surface. An ascending psychophysical method of limits was used. The mean of three millivolt readings was accepted as the lingual threshold.

Subsequent to threshold acquisition, DLs were determined at five sensation levels $(10,15,20,25$, and $30 \mathrm{mV} \mathrm{SL})$ for each of the three test frequencies. For each frequency, a subject was presented one of the sensation-level stimuli. The subject was instructed to concentrate on the intensity of the stimulus and 


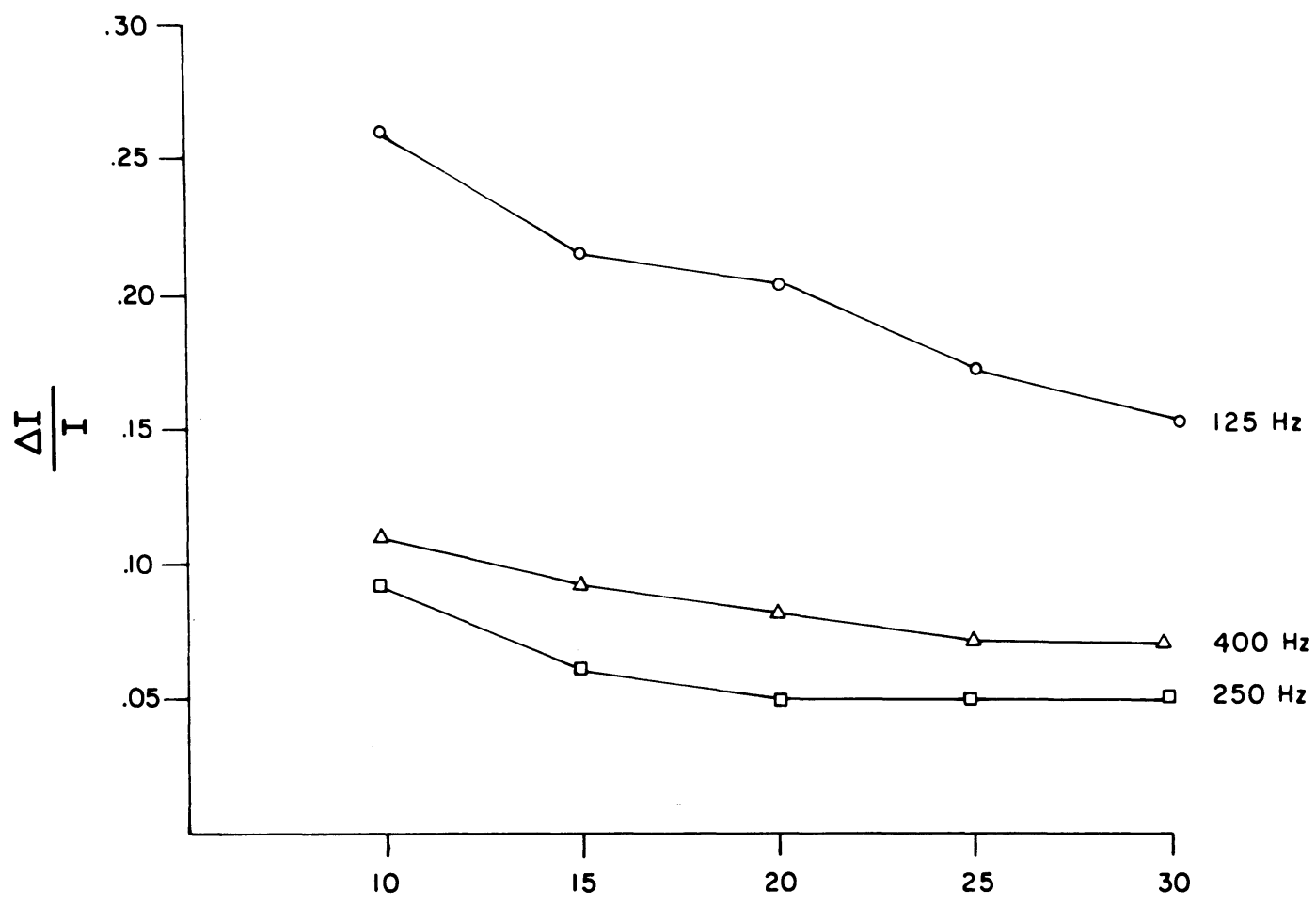

\section{Sensation Levels in Millivolts}

Figure 1. Lingual vibrotactile difference-limen intensity values for five sensation levels at three different frequencies.

to make a "mental set" of that sensation. When the reference signal was internalized, a comparison signal, varying only in intensity, was delivered to the lingual surface. The subject's task was to indicate whether the second stimulus was the same as or different from the reference stimulus. This procedure was repeated until DLs were achieved for each of the five sensation levels at each of the three frequencies. A bracketing procedure was used to obtain the DLs. This procedure involved the determination of the jnd in . $10-\mathrm{dB}$ steps. A similar memory task methodology was utilized by Harris (1963) for obtaining DLs of intensity in the human auditory system.

\section{RESULTS AND DISCUSSION}

Figure 1, shows the mean DL values for the five subjects as a function of frequency and sensation level. With respect to the frequency parameter, it can be seen that the DL values for 250 and $400 \mathrm{~Hz}$ are somewhat similar for all sensation levels tested. The DL values for $125 \mathrm{~Hz}$ are greater than those for 250 and $400 \mathrm{~Hz}$ for all sensation levels tested. There is also a trend at $125 \mathrm{~Hz}$ for larger $\mathrm{DL}$ values to occur at the lower sensation levels.

The results of this investigation are not in complete agreement with those described for the auditory sensory system. Unlike the DL values for the auditory system, those for the lingual tactile system appear to be frequency dependent, at least in terms of the three fre- quencies investigated. The lingual DL values for $125 \mathrm{~Hz}$ are seen to be higher than those for 250 and $400 \mathrm{~Hz}$ (Figure 1). There also appears to be a slight dependence on sensation level for the lingual tactile sensory system. The lower sensation levels (those nearest threshold) yield somewhat larger DL values, especially at $125 \mathrm{~Hz}$. This sensation-level dependency is also reported for the auditory sensory system (Harris, 1963; Riesz, 1928).

The lingual DL results appear to be compatible with those found on the hand and index finger, in that lower sensation levels (those nearest threshold) provided larger DL values (Craig, 1972). In terms of frequency, however, the DLs derived from the hand and index finger are frequency independent, whereas those for the tongue tend to show frequency dependence for the range of frequencies studied.

It is very difficult to make any kind of accurate comparisons of the findings of this study with those of any of the other investigations concerned with DL values. Irrespective of the modality being investigated, each individual study appears to employ some differences in methodologies, instrumentation, and data plotting techniques. The most that can be done is to study trends and identify similarities and differences. Overall, the data obtained from this study indicate that it is possible to obtain DL functions from the lingual sensory system. It can provide functions that are reason- 
ably in line with those of other tactile sensory systems, as well as those derived from other sensory modalities, such as audition. Confirmation of these observations and conclusions awaits additional research in this area.

\section{REFERENCES}

Craig, J. C. Difference threshold for intensity of tactile stimuli. Perception \& Psychophysics, 1972, 11, 150-152.

FuCCI, D., \& CRARY, M. Oral vibrotactile sensation and perception: State of the art. In N. J. Lass (Ed.), Speech and language: Advances in basic research and practice (Vol. 2). New York: Academic Press, 1979.
Harris, J. D. Loudness discrimination. Journal of Speech and Hearing Disorders, Monograph Supplement II, 1963.

KNuDsEN, V. D. Hearing with the sense of touch. Journal of General Psychology, 1928, 1, 320-352.

Riesz, R. R. Differential intensity sensitivity of the ear for pure tones. Physics Review, 1928, 31, 867-875.

Schiller, H. Über die Amplitudenunterschiedschwellen des Vibrationssiness beim Menschen. Unpublished doctoral dissertation, University of Erlangen, 1953.

Sherrick, C. E. Measurement of the differential sensitivity of the human skin to mechanical vibration. Unpublished master's thesis, University of Virginia, 1950.

(Received for publication April 29, 1982.) 